\title{
Construction of 3D Model of Tunnel Based on 3D Laser and Tilt Photography
}

\author{
Yuan-Rong He, ${ }^{1}$ Ping Chen, ${ }^{*}$ Wei-Wei Ma, ${ }^{1}$ and Chih-Cheng Chen ${ }^{2 *}$ \\ ${ }^{1}$ Xiamen University of Technology, College of Computer and Information Engineering, \\ Xiamen, Fujian 361024, China \\ ${ }^{2}$ Jimei University, School of Information Engineering, Xiamen, Fujian 361021, China
}

(Received October 14, 2019; accepted February 17, 2020)

Keywords: laser point cloud, high-speed tunnel, tilt photography, information acquisition, 3D modeling

In this study, we take a section of a tunnel located on the expressway as the research subject and propose a 3D model based on high-precision and high-spatial-resolution 3D laser scanning technology to obtain tunnel point cloud data information. The tunnel is built using unmanned aerial vehicle (UAV) tilt photogrammetry technology. The model finally realizes the combination of the 3D visual model of the tunnel and the 3D space around the tunnel. The Trimble TX8 ground 3D laser scanner is used as the tunnel 3D point cloud data acquisition device. Through 3D laser scanning, the collected 3D point cloud is processed by data sampling, thinning filtering, registration, and splicing. With the algorithm of point cloud data to process the fitting and repair of the point cloud, the tunnel information data is effectively acquired and the 3D model is constructed. The construction of a 3D model is completed on the basis of the same phase control points of oblique photography acquisition. The analysis results show that the 3D laser scanning technology has clear effects on the extraction of tunnel spatial data and the construction of the model. The effective combination of 3D laser scanning technology and tilt photography technology has improved the quality of 3D model construction in urban areas and is a major breakthrough in the field of 3D modeling.

\section{Introduction}

Terrestrial laser scanning (TLS) is an advanced technology that began to appear in the mid1990s. The sensor used by the 3D laser scanner is a high-speed and accurate laser rangefinder, coupled with a set of reflective prisms that can guide the laser and scan at a uniform angular velocity. It can obtain the 3D information of objects quickly without contact by laser ranging. ${ }^{(1)}$ The measurement principle is that the laser rangefinder actively emits laser light and simultaneously receives the signal reflected from the surface of the natural object, so that the distance can be measured. For each scanning point, the slope distance from the measuring station to the scanning point can be measured. The direction angle can obtain the spatial relative

*Corresponding author: e-mail: chtchenping@163.com

**Corresponding author: e-mail: 201761000018@jimu.edu.cn

https://doi.org/10.18494/SAM.2020.2692 
coordinates of each scanning point and the station. If the spatial coordinates of the station are known, the 3D coordinates of each scan point can be obtained. TLS has broken through the traditional measurement method and has the advantages of noncontact, high efficiency, and high precision to obtain 3D point cloud data on the surface of the object, and it has a farreaching development prospect in many fields. 3D laser scanning technology can realize a fast and accurate 3D model construction for various large-scale irregular, complex buildings and environments with insufficient light, which provides an important technical guarantee for complex construction engineering design. ${ }^{(2)}$ Because of the poor lighting conditions in tunnels, narrow internal spaces, and many interference factors, traditional measurement methods have many problems, such as long collection operation time and high labor cost, and it is very difficult to establish a 3D model. Laser scanning technology frees the acquisition and detection of tunnel spatial information from the traditional bottleneck. It has the unique advantages of fast data acquisition, large amount of data acquisition, strong real-time performance, visual processing, clear object texture and details, convenient operation, and so forth. ${ }^{(3)}$ These features will be widely used in the 3D reconstruction of various architectural fields, especially in the area of tunnel modeling. Tilt photogrammetry is a new type of aerial photography, which uses a tilt camera mounted on an aerocraft to obtain object information. The aerocraft can carry multiple sensors in the same flight platform. The multiple sensors can capture images from 5 angles such as 1 vertical downward and 4 angles of 45 degrees downwards to obtain the real object information, and the post-processing software of Street View Factory is used to construct a real-life 3D model, which is applied to the 3D construction of smart cities. ${ }^{(4)}$

With the development of science and technology, people have higher requirements on the measurement accuracy, resolution, and definition of the real-scene 3D model. The emergence of the 3D laser has accelerated the development of engineering information, and the point cloud data is very practical after processing. With the continuous improvement of the measurement accuracy of the 3D laser scanner, its application range has gradually expanded to all types of industries in architecture. Moreover, there is a breakthrough in obtaining architectural information by leaps and bounds. ${ }^{(5)}$ On the basis of the high-precision measurement, the complete 3D data of the tunnel can be obtained in the form of a true color point cloud image. The registration accuracy is improved by marking the target sphere, extracting the feature point cloud, measuring the tunnel spatial information, and effectively extracting the texture information for the point cloud tunnel model. ${ }^{(6)}$ Because of the large amount of data collected by the 3D laser, it takes much time to process the data. The tilt photography modeling of an unmanned aerial vehicle (UAV) has the characteristics of high efficiency, high speed, and vivid color effect. In this study, two technologies are combined to complement each other. In some tunnel information that requires high-precision modeling and processing, the 3D modeling of the point cloud is adopted. The UAV is equipped with a high-definition camera around the tunnel to perform tilt photography. The research results show that the laser scanning technology is effective in the construction of the 3D tunnel model, and that the combination of laser scanning and tilt photography improves the quality of the model and has made a certain breakthrough in the field of architecture. 


\section{Tunnel Data Acquisition}

The scanning tunnel is located on a highway section with a length of $400 \mathrm{~m}$. The Trimble TX8 3D laser scanner is used for scanning, and the high measurement precision of TX 8 can be kept within the range of $120 \mathrm{~m}$ around it. Roadblocks should be assumed during scanning to ensure the safety of personnel and equipment. To obtain all-directional and high-precision 3D tunnel data, instrument scanning should have high resolution, high quality, and color scanning. In the scanning, it is necessary to appropriately control the setting interval of the scanner to ensure the measurement accuracy and integrity of the point cloud. If the distance between the stations is large, the number of stations will be small and the measurement time will be shortened, but the quality of the point cloud will decrease when the distance increases. ${ }^{(7)}$ The distance between the stations is controlled within $30 \mathrm{~m}$, taking into account the measurement efficiency and point cloud quality. To obtain the complete point cloud of the end wall of the tunnel, one station is added to the outside of the tunnel entrance and exit. A total of 15 scanning stations are set up. The scanning time of each station is $5 \mathrm{~min}$, so as to ensure the integrity of the information. Considering that the tunnel with two lanes passing in this section is a circular shield tunnel, the right lane is closed when data are collected, the center side of the tunnel is set up on the right side of the tunnel, and the height scanner is appropriately set up during scanning to reduce the interference of traffic. The scanning process mainly includes instrument parameter debugging, control measurement, control point layout, target cloth measurement, station scanning, data quality inspection, and data export backup. ${ }^{(8)}$ The laser scanning scene is shown in Fig. 1.

\section{Preprocessing of Point Cloud Data}

The collected point cloud data are imported into Trimble RealWorks software for data preprocessing. Firstly, the point cloud data should be sampled and processed. In this work, step sampling is used. If the step size is 2 , the total number of points after sampling is $1 / 4$. If the

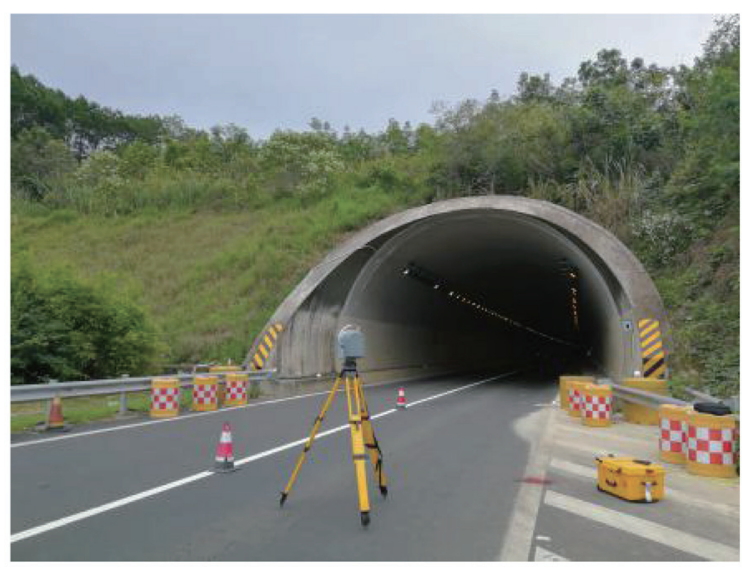

Fig. 1. (Color online) Field scan. 
step size is 3 , the total number of points after sampling is $1 / 9$. To restore the real model as much as possible and obtain complete data for high-precision measurement and monitoring, the tunnel point cloud data packet obtained by sampling data with a step size of 1 contains a large amount of noise, which should be preprocessed. ${ }^{(9)}$ The preprocessing includes the thinning filtering of point cloud data, splicing registration, curved surface fitting, coordinate transformation, then extracting the spatial information, combining with the 3D model established by tilt photography, and finally, outputting the results. The specific technical route is shown in Fig. 2.

\subsection{Data extraction and filtering}

After sampling the point cloud data, the total number of point clouds is as high as 1.6 billion, and the data size is as large as $8 \mathrm{~GB}$. Because of the large amount of data, the point cloud data should be extracted and filtered before stitching, so as to reduce the data capacity and improve the efficiency of data mosaic registration. The random sampling method is used in this study. The result of random sampling is that the dense part of the point cloud is still relatively dense after sampling, and the place where the point cloud is sparse will be more sparse. After extracting, the details of the tunnel can still be completely preserved. ${ }^{(10)}$

Since the tunnel is located on a traffic-intensive highway, blocking and obscuring the scanning target, the overall point cloud contains much redundant data, which makes the laser scanning more difficult and requires noise deletion. Automatic filtering can be carried out by point cloud distance and area filtering. However, there may be some problems, such as the accidental deletion of the tunnel point cloud and the incomplete removal of unwanted noise. According to the contour of the tunnel model, the data is filtered by manually extracting noise. Tunnel point clouds are cut in layers by using different perspectives to remove the interfering point cloud noise. ${ }^{(1)}$ After the extraction and drying, the total number of point clouds is 1.3 billion and the data size is $6 \mathrm{~GB}$; thus, the registration efficiency can be considerably improved on the premise of ensuring the integrity of the tunnel model after stitching.

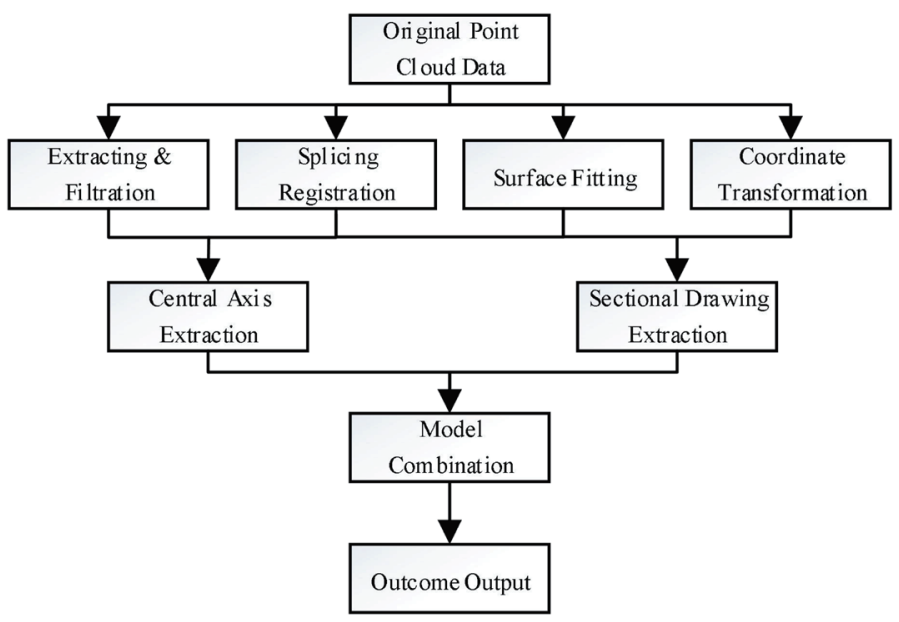

Fig. 2. Technical route of data processing. 


\subsection{Point cloud data registration}

At present, there are three methods of point cloud data registration, i.e., automatic extraction of target (target ball and target paper) automatic registration, nontarget automatic registration, and manual registration. ${ }^{(12)}$ Because the overall surface of the tunnel is relatively smooth and the structure is symmetrical, the point cloud is difficult to match automatically. It is easy to cause the mismatch of several adjacent survey stations and the splicing of the intersection in and out of the two tunnels owing to the high similarity. However, there are some problems in automatic target extraction, such as insufficient light intensity in the tunnel, noise produced by vehicles passing through to block the target ball, and low precision of automatic target ball recognition and extraction.

To solve some problems in the above registration methods, in this study, we propose a method of combining a manual target and extracting a feature point cloud to carry out the registration of point cloud data. On the basis of the target ball registration, at least three target balls are selected in the scanning point cloud of different stations that should be registered, with manual marking as the main registration method. The target ball may be blocked by interference noise such as that produced by vehicles at tunnel crossings. There are problems such as low stitching accuracy and registration failure. The method of extracting the feature points of two adjacent stations combined with the target ball is used to extract the common feature points of two stations as the reference points for registration, ensuring that there are three or more reference points. The target ball is selected by the panoramic plane view scanned by each station, and the feature point is marked by selecting the same feature point in the overlapping area of two adjacent stations through the $3 \mathrm{D}$ view. The target ball marker is shown in Fig. 3.

After all the data points are marked, the point cloud splicing can be realized, and the accuracy of point cloud data registration should be checked. To integrate the two models accurately, the precision error of the selecting target ball and marking points should be controlled within $1 \mathrm{~mm}$. After registration, the maximum error of the overall data is within $2 \mathrm{~mm}$ and the minimum error is $0 \mathrm{~mm}$. After stitching, the tunnel point cloud model is rendered in true color, and the complete 3D tunnel model is restored, as shown in Fig. 4.

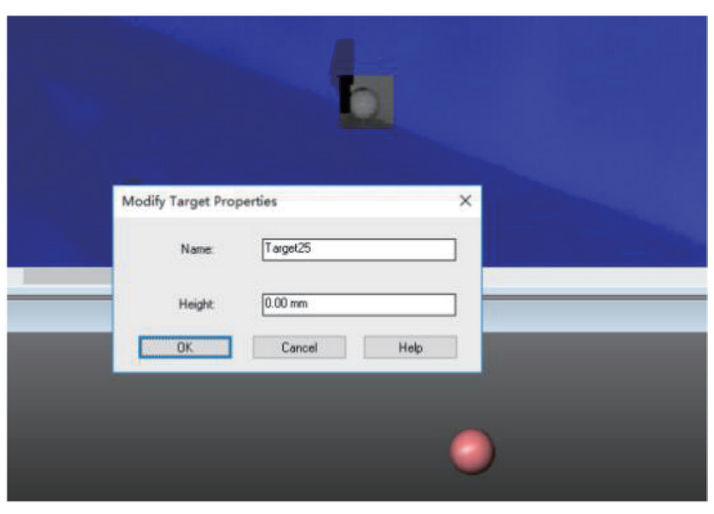

(a)

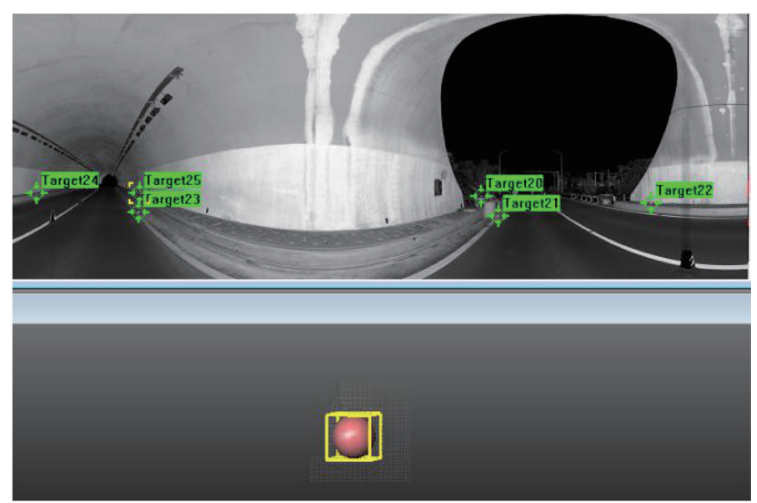

(b)

Fig. 3. (Color online) Target ball marker. (a) Selected target ball. (b) Marked target ball. 


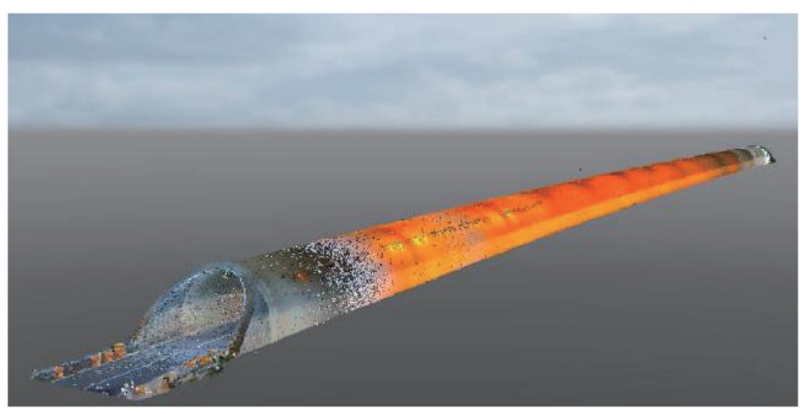

Fig. 4. (Color online) 3D tunnel model.

\subsection{Tunnel surface fitting}

Owing to the insufficient tunnel light and vehicle interference, the generated point cloud data contains the area that cannot be scanned by the point cloud, that is, part of the cavity exists. Point cloud holes are the missing point clouds caused by the obstruction of 3D laser scanning. The point cloud holes will have a considerable impact on the subsequent processing of the point cloud, so there are generally two ways of repairing the point cloud holes: (1) in the field supplementary measurement, the basic principle is to scan or measure the hole area of the point cloud using a 3D laser scanner or an electronic total station, and to match the obtained data to the point cloud data. (2) The point cloud interpolation method is based on the point cloud data around the cavity, combined with the field photos, and the algorithm in the data processing software is used to interpolate and repair the cavity on the computer. When selecting the software algorithm, we should combine the actual terrain with the cavity area terrain to select the appropriate algorithm for interpolation. ${ }^{(13)}$ Because of the particularity of the management of high-speed tunnel sections, the secondary acquisition of field compensation and transmission will have some effects, such as traffic congestion, so the point cloud interpolation method is used to repair it.

\subsection{Transformation of coordinate system}

Since tilt photogrammetry and 3D laser scanning are two sets of relatively independent measurement technology systems, the measurement results have their own independent coordinate systems. The point cloud acquired by different stations should be placed in a unified coordinate system, and coordinate transformation is performed according to the direction of the tunnel axis, so that one coordinate axis is aligned with the tunnel axis direction, and the coordinate origin is located on the tunnel axis. 3D laser scanning measurement is generally an instrument self-defined coordinate system. The $X$-axis is in the lateral scanning plane, the $Y$-axis is perpendicular to the $X$-axis in the lateral scanning plane, and the $Z$-axis is perpendicular to the lateral scanning plane. The calculation principle of the scanning measurement coordinate $P$ is shown in Fig. 5.

The data directly scanned by the scanner is based on the scanning coordinate system of the scanner. The national or independent coordinate system often used in the cloud is transformed 


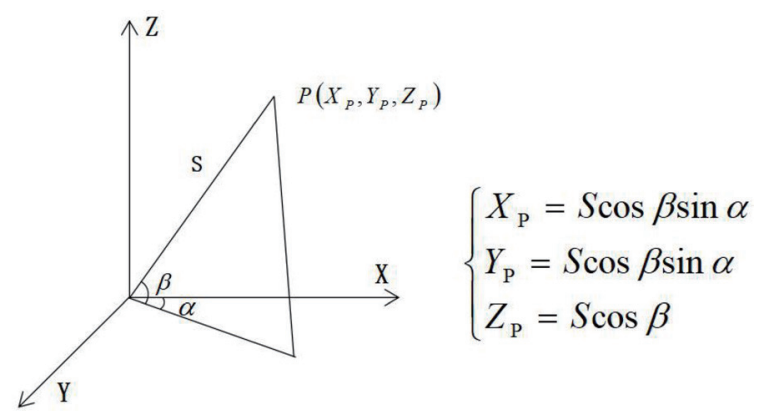

Fig. 5. Principle of coordinate calculation of scanning points.

from the scanner coordinate system to the practical coordinate system actually required, and the phase control points are collected by real-time kinematic (RTK) and the phase control points are selected in the visible area during laser scanning. The coordinate information can be collected by selecting the surrounding feature points or the middle points of road painting. The coordinates are established in the postprocessing software Trimble RealWorks. The coordinate system of the initial point cloud is only an assumed coordinate system, and RTK can set the absolute coordinate system we need as a national coordinate system or a local independent coordinate system. Therefore, the phased points marked in the point cloud model are selected and the coordinate values measured by the RTK are assigned at those points. After all the assignments are completed, all points of the point cloud model are located in the same coordinate system. The main method is implemented by the transformation matrix. After the transformation, the error of the phase control point is controlled within $5 \mathrm{~mm}$. As shown in Fig. 6, the coordinate error before and after the conversion is $2 \mathrm{~mm}$, which meets the requirements and ensures the accuracy of the two models in the later pairing.

\section{Extraction of Spatial Information}

According to the processed 3D point cloud, the complete geometric model of tunnel engineering can be established. In the stage of spatial information analysis, we can extract the central axis and analyze the geometry of the sectional graph. According to the characteristics of the tunnel, each part of the processing can be divided into sections, so as to ensure the continuity between the sections. The analysis can be displayed in two different ways by software, such as 2D and 3D.

\subsection{Extraction of central axis}

The central axis of the tunnel reflects the direction and attitude of the tunnel as a whole, and extracting the central axis can obtain more complete tunnel information. ${ }^{(14)}$ The method of extracting the axis is to fit the curved circle of the tunnel point cloud, and the axis of the curved circle is taken as the axis of the tunnel. Then, the tunnel is divided into sections, the alignment of the cylinder is close to the standard circle, and the axis of the cylindrical surface is regarded as the axis of each section of the tunnel. In view of the characteristics of the cloud 


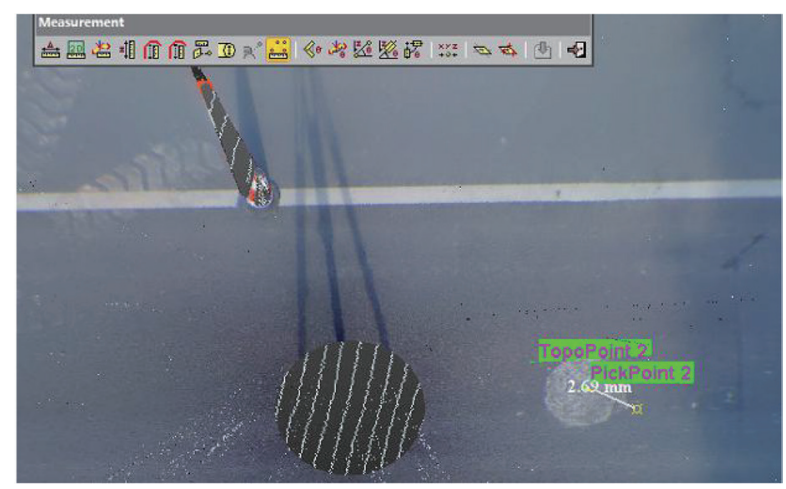

Fig. 6. (Color online) Coordinate conversion accuracy.

band distribution of tunnel points, it is proposed that the azimuth maximum value search algorithm is used to extract edge points to fit the central axis of the tunnel. ${ }^{(15)}$ To improve the efficiency of fitting, we shall sample and dilute the point cloud data, and then use the Geomagic software and manual interaction methods to further filter connection bolt holes, nuts, grouting holes and cables, lighting equipment, and other facilities attached to the pipe wall in the tunnel point cloud. To ensure that the searched points were located on the inner wall of the tunnel as far as possible, the original point cloud was projected onto the horizontal plane (XOY) after filtering and planarly rotated to make the tunnel trend consistent with the $X$-axis. The central axis of the tunnel can be obtained from the average of the upper and lower two edge lines. The azimuth starting direction of the upper edge point is the positive direction of the $Y$-axis, and the starting direction of the azimuth angle is the reverse direction of the $Y$-axis in the search for the lower edge. A seed point is chosen from the tunnel point cloud, the search radius $r$ is set, the azimuth of each point and that of the seed point within the search radius are calculated, and the maximum or minimum azimuth point is selected as the seed point for the next search. If the minimum azimuth is used as the search condition to indicate the search to the right and if the search is performed to the left side, the point set of the two-direction search constitutes a complete edge line. ${ }^{(16)}$

\subsection{Drawing of 2D section}

The tunnel cross-sectional view refers to a series of vertical cross sections of the tunnel perpendicular to the center line of the tunnel, which represents the geometric shape of the closed intersecting line between the inner surface of the tunnel and the vertical section. Its horizontal and vertical coordinates respectively represent the horizontal and vertical coordinates of the points on the intersecting line on the vertical section. ${ }^{(17)}$ A cross-sectional view of the tunnel is drawn and a 2D line drawing of the point cloud is extracted. With this, the structure and geometry information of the tunnel can be obtained from the spatial information inside the tunnel on both sides.

To establish a section at any point on the tunnel wall, the corresponding point on the central axis must be found first, and the minimum distance between the two must be satisfied. For any point $P\left(x_{p}, y_{p}, z_{p}\right)$ on the wall of the tunnel, search first the central axis for the corresponding 
point $p\left(x_{p}, y_{p}, z_{p}\right)$, so that the distance between them is minimized, that is, the distance between them is satisfied.

$$
p=\left\{p_{i} \in f, g \mid \sqrt{\left(x_{P}-x_{p_{i}}\right)^{2}+\left(y_{P}-y_{p_{i}}\right)^{2}+\left(z_{P}-z_{p_{i}}\right)^{2}}=\min \right\}
$$

In the formula, $p_{i}$ is the set of $p$ points on the central axis $i=1,2, \ldots, n . f \mathrm{~g}$ is the equation of the tunnel centerline, which is obtained by fitting $y$ and $x, z$ to the quadratic curve, respectively, and can be expressed as $f=x_{p}=a_{0}+a_{1} y_{p}+a_{2} y_{p}^{2}, g=z_{p}=b_{0}+b_{1} y_{p}+b_{2} y_{p}^{2}$. The plane equation perpendicular to the tangent line of $P$ can be expressed as

$$
y+k_{p} \cdot x+k_{p}^{\prime} \cdot z+m=0
$$

where $\left(1, k_{p}, k_{p}^{\prime}\right)$ is the instantaneous tangent vector at point $p$, which can be calculated according to Eq. (3), that is,

$$
k_{p}=\left.\frac{d x}{d y}\right|_{y=y_{p}}, k_{p}^{\prime}=\left.\frac{d x}{d y}\right|_{y=y_{p}} .
$$

If the thickness of the section is $d$, the point set $Q$ within the range is defined as

$$
Q=\left\{P_{i} \in P|| \frac{y_{i}+k_{i} \cdot x_{i}+k_{i}^{\prime} \cdot z_{i}+m}{\sqrt{1+k_{i}^{2}+k_{i}^{\prime 2}}} \mid \leq \frac{d}{2}\right\}
$$

In this study, the cross-cutting technique is used to extract the tunnel point cloud section in the Trimble RealWorks software by cutting the central axis, and the contour of the point cloud is obtained. ${ }^{(18)}$ Because the tunnel can be captured at every point, it is possible to create a line graph reflecting the physical information characteristics of the tunnel according to the point cloud and automatically fit it into a line. By fitting the 2D line, an accurate tunnel leveling

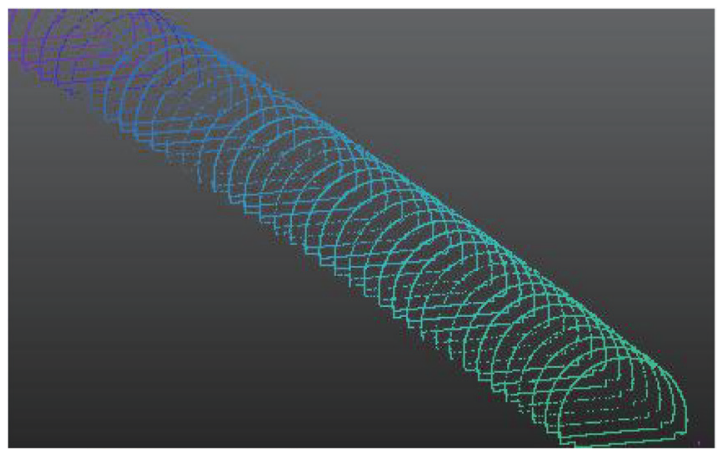

(a)

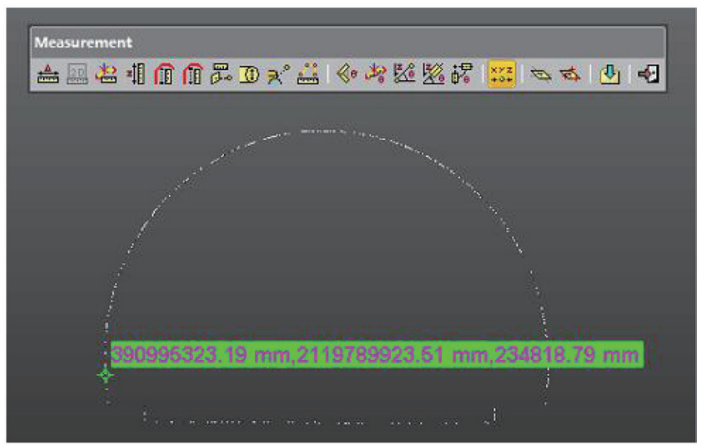

(b)

Fig. 7. (Color online) Tunnel section. (a) Overall section. (b) Partial section. 
profile can be obtained. The overall shape of the tunnel is the upper part of the circle. After the interception section is completed, the intercept points on each section can be connected separately, and each section forms a separate ring and is displayed in 3D. The extracted tunnel point cloud cross-sectional view is shown in Fig. 7, including the inner wall of the tunnel, the inner overhaul path, and the road. The distance between points and the $3 \mathrm{D}$ coordinates of points can be measured in the section diagram.

\section{Combination of 3D Models}

UAV tilt photography technology is utilized to measure the tilt photos of the tunnel entrance and surroundings to generate a 3D tilt model outside the tunnel. The point cloud data of the tunnel is imported into the tilt model, and the unified coordinate system of CGCS2000 is used to combine the two models to complete the construction of the tunnel panorama model.

\subsection{Generating the tilt model of tunnel}

The image data processing software collected by UAV adopts the real-scene modeling software Context Capture, which can use the image data captured by various digital cameras to easily generate the real-time 3D model with high resolution for the required environment. By using any ruler or resolution to create the required 3D model, with digital photo textures and geographic location based on a coordinate system, the model products are produced directly from the image data. ${ }^{(19)}$ There are four main points for establishing a 3D model by Context Capture Master: (1) loading aerial image photos, (2) importing the image control point coordinate file and adding the control point, (3) calculating the triangulation in air, and (4) constructing the 3D model full automation.

The UAV 3D point cloud is a collection of data that records the color and elevation information of the surface in the form of discrete points, which can be used to perform complete 3D surface modeling and realize 3D visualization. It can also be used to calculate the stereoscopic 3D parameters, including the slanting distance of the surface, the height of the deep pit, and the volume of the bulk of loose objects. The tunnel point cloud data is imported into the tilt model, and the panoramic model is constructed using the unified coordinate system of CGCS2000, as shown in Fig. 8.

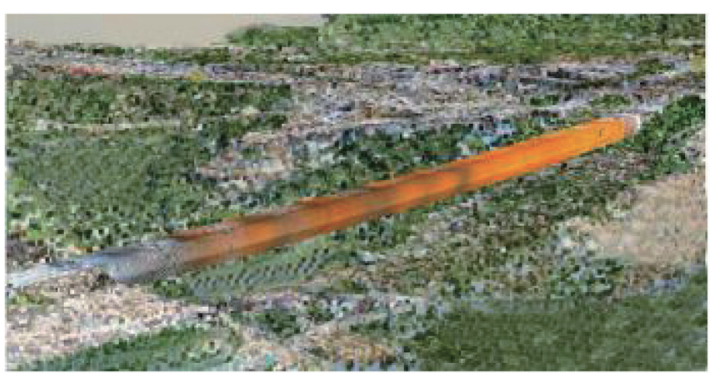

(a)

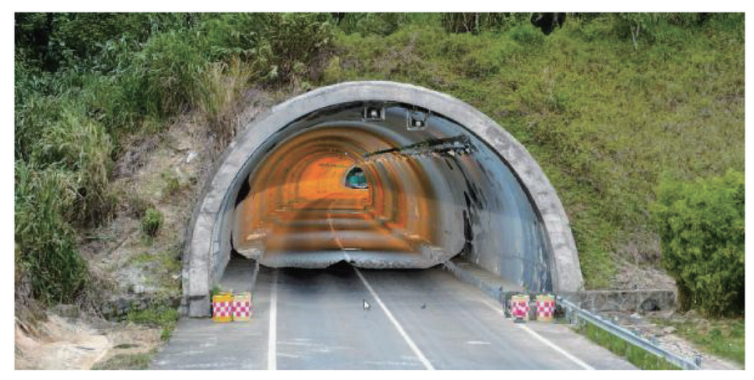

(b)

Fig. 8. (Color online) Panoramic 3D model. (a) Panoramic model. (b) Tunnel front view. 
Table 1

Comparison with other measurement methods.

\begin{tabular}{lccc}
\hline Methods & $\begin{array}{c}\text { Model inside } \\
\text { the tunnel }\end{array}$ & $\begin{array}{c}\text { Out-of-tunnel } \\
\text { model }\end{array}$ & $\begin{array}{c}\text { Tunnel panorama } \\
\text { model }\end{array}$ \\
\hline 3D laser and tilt photogrammetry of UAV & $\sqrt{ }$ & $\sqrt{ }$ & $\sqrt{ }$ \\
GPS system & $\times$ & $\times$ & $\times$ \\
3D laser & $\sqrt{ }$ & $\times$ & $\times$ \\
Tilt photogrammetry of UAV & $\times$ & $\sqrt{ }$ & $\times$ \\
3D max modeling technology & $\sqrt{ }$ & $\times$ & $\times$ \\
Virtual simulation modeling technology & $\sqrt{ }$ & $\times$ & $\times$ \\
\hline
\end{tabular}

It can be seen from Fig. 8 that the coordinated cooperation between the 3D laser sensor and the UAV tilt photography sensor is the biggest novelty of this research. A panoramic model of the tunnel is formed by combining the 3D model data of the point cloud inside the tunnel with the tilt model outside the tunnel. The tilt model outside the tunnel obtains highprecision, high-resolution texture information, which can be used for tunnel opening planning and subsequent analysis of the slope danger. The 3D model of the point cloud inside the tunnel provides powerful data support for subsequent tunnel measurement, monitoring, 2D crosssectional drawing, tunnel management and maintenance, and other tasks. This model improves the quality of 3D models of urban construction and is a major breakthrough in the field of 3D modeling.

\subsection{Comparison with other measurement methods}

Earlier research on tunnel measurement mainly used the GPS system as a measurement method to obtain the GPS control network of the tunnel, but it was difficult to generate a 3D model of the tunnel. ${ }^{(20)}$ With the development of 3D modeling technology, the 3D modeling technology of tunnels has been studied by many researchers, such as those whose interests are in 3D laser scanning technology to monitor the tunnel, ${ }^{(21)}$ drones to collect high-definition images of tunnel openings and build tunnel opening models, ${ }^{(22)} 3 \mathrm{D}$ max software to structure the $3 \mathrm{D}$ tunnel models, ${ }^{(23)}$ and simulation technology to construct $3 \mathrm{D}$ tunnel visualization models. $^{(24)}$ Table 1 shows the comparison between this study and other tunnel measurement methods.

From Table 1, it can be seen that the 3D laser and UAV tilt photography technology used in this study can obtain a panoramic model of the tunnel. In addition, no other method has a panoramic model. UAV photography technology cannot obtain the 3D data inside the tunnel. Only a 3D laser, 3Dmax modeling technology, and virtual simulation modeling technology can obtain 3D data inside the tunnel.

\section{Conclusion}

In this study, the advantages of 3D laser scanning technology combined with the tilt photogrammetry of UAV are used to solve the problems of local detail complementation and fine modeling of tilt photography by using high-precision point cloud data collected with a 3D 
laser. The 3D modeling of tilt photography has high efficiency and good effect, which solves the problem of low modeling efficiency caused by the huge amount of point cloud data. The control point coordinate system of RTK unified laser scanning and tilt photography ensures the uniformity of the coordinates of the two measurement results. Compared with other research methods, the combination of the two technologies has enriched the means of modeling data collection and improved the quality of the city's 3D model. It can also build a panoramic model of the tunnel, which meets the project's requirements for the construction of various data 3D models. High-speed tunnel construction provides better technical support.

3D laser scanning technology and oblique photogrammetry are advanced technologies in the field of surveying and mapping. With the continuous construction and development of highways, viaducts, and tunnels, and the continuous increase in traffic volume, the combination of the two technologies can efficiently and widely acquire 3D spatial information, and multiangle image information will definitely make important contributions in the field of 3D modeling. In the future, the measures to ensure a high detection accuracy can be further improved in practice, and the modeling efficiency can be optimized in a continuous manner. Finally, the acquisition accuracy and modeling speed can be further improved on the existing basis, so as to form a set of rapid and effective mature detection systems.

\section{Acknowledgments}

This research was supported by the Science and Technology Planing Foreign Cooperation Project of Longyan (Grant No. 2018LYF7006) and Shanxi Key Laboratory of Resources, Environment and Disaster Monitoring (Grant No. 2019-02).

\section{References}

1 H. Shi: Study on the Application of 3D Laser Scanning Technology in the Monitoring of Construction Tunnel (Beijing Jiaotong University, Beijing, 2013).

2 Y. Lu: Application Research of Bridge Detection Technology Based on 3D Laser Scanning (Jilin University, Changchun, 2017).

3 L. Yang: Study on Application of 3D Laser Scanning Technology in Deformation Monitoring in Construction of Building Engineering (Tianjin University, Tianjin, 2017).

4 R. C. Tan, P. P. Li, L. Wen, and C. Pan: Surv. Mapp. Bull. (2016) 61. https://doi.org/10.13474/ j.cnki.11-2246.2016.0361

5 Y. R. He, H. P. Pan, Y. M. Zheng, and W. C. He: Laser J. 37 (2016) 90. https://doi.org/10.14016/j.cnki. jgzz.2016.02.090

6 X. Y. Xie, X. Z. Lu, H. Y. Tian, Q. Q. Ji, and P. Li: Surv. Mapp. Bull. (2016) 143.

7 H. X. Yu: Modeling of Complex Buildings Based on 3D Laser Scanning Measurement Technology (China University of Mining and Technology, Xuzhou, 2014).

8 M. Wang, J. Feng, and Z. H. Xie: Bull. Surv. Mapp. (2014) 78. https://doi.org/10.13474/j.cnki.11-2246.2014.0403

9 F. R. Gui: Bullet. Sci. Technol. 31 (2015) 263. https://doi.org/10.13774/j.cnki.kjtb.2015.12.094

10 H. F. Xing, Z. G. Gao, and L. Lu: Eng. Invest. Survey. 42 (2014) 52.

11 H. Xu: Application of Close-Aange Photogrammetry and 3D Laser Scanning in Tunnel Deformation Monitoring (Jilin Architecture and Civil Engineering Institute, Changchun, 2016).

12 L. Peng, Z. H. Huang, and P. Cheng: Survey. Map. Bull. (2017) 163.

13 Q. P. Xie, H. Y. Yu, P. L. Yu, X. P. Lu, and C. Li: Sci. Surv. Mapp. 40 (2015) 98. https://doi.org/10.16251/ j.cnki.1009-2307.2015.06.0020

14 Y. Lu: Application Research of Bridge Detection Technology Based on 3D Laser Scanning (Jilin University, Changchun, 2017). 
15 Y. B. Lin: Application Research of 3D Lidar Image in Railway Foreign Body Invasion Detection (North China University of Technology, Beijing, 2018).

16 C. Li: Bull. Surv. Mapp. 11 (2012) 98.

17 Y. R. He, Y. M. Zheng, H. P. Pan, and J. Z. Chen: Remote. Sens. Tech. Appl. 31 (2016) 1091. https://doi. org/10.11873/j.issn.100-0323.2016.6.1091

18 Q. Y. Xie: Surv. Mapp. 39 (2016) 138. https://doi.org/10.3969/j.issn.1674-5019.2016.03.012

19 G. C. Ma, L. J. Wang, S. Ma, and H. Liu: J. Safe. Sci. Tech. 13 (2017) 73. https://doi.org/10.11731/j.issn.1673193x.2017.05.012

20 X. D. Zhang and Z. L. Zhang: Invest. Tech. 18 (2001) 85. https://doi.org/10.3969/j.issn.1001-3946.2000.05.010

21 X. J. You, and D. F. Zhan: Surv. Mapp. Bull. (2017) 80. https://doi.org/10.13474/j.cnki.11-2246.2017.0125

22 M. Ma, J. B. Zhu, B. H. Zhan, B. Lu, C. Li, B. Wang, Y. Z. Jiang, X. H. Liu, and D. D. Xu: Transp. Tech. 44 (2019) 98.

23 Y. F. Chen, S. Cao, F. Li, Z. D. Liu, J. C. Chen, and Y. J. Qin: Comput. Knowl. Tech. 14 (2018) 262.

24 D. H. Zhong, K. J. Liu, and K. X. Wu: J. Eng. Graphics 26 (2005) 81. https://doi.org/10.3969/ j.issn.1003-0158.2005.04.016

\section{About the Authors}

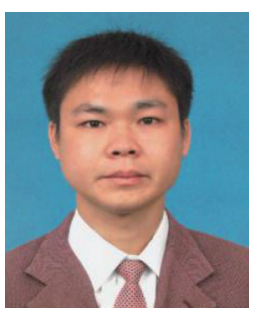

Yuan-Rong He received his bachelor's and master's degrees from Guilin University of Science and Technology in 1997 and 2004, respectively. In 2011, he received his Ph.D. degree from Central South University in China. From 2014 to 2017, he conducted postdoctoral research at the Institute of Urban Environment, Chinese Academy of Sciences. He has been a professor of Xiamen University of Technology since 2012. His research interests are in multisensor integrated systems based on GIS. (2012112001@xmut.edu.cn)

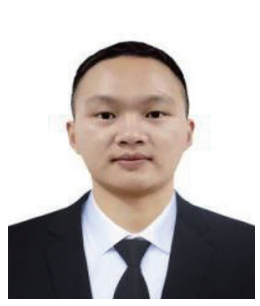

Ping Chen received his B.S. degree from Xiamen University of Technology, China, in 2018. Since 2019, he has been a graduate student at Xiamen University of Technology, China. His research interests are in 3D laser scanning, data integration, and visualization. (chtchenping@163.com)

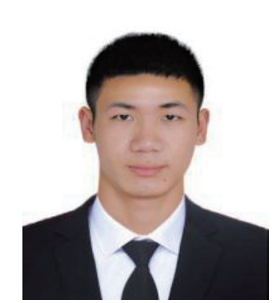

Wei-Wei Ma received his B.S. degree from Fujian Agricultural and Forestry University in China in 2017 and studied for his M.S. degree at Xiamen University of Technology in China from 2017 to 2020. His research interests include drone technology and laser-point cloud technology.

(maweiwei1019@gmail.com)

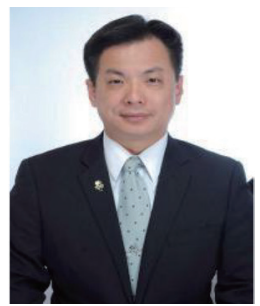

Chih-Cheng Chen has been a professor at Jimei University, China, since 2017. He became a member of IEEE in 2011 and a senior member in 2016. He earned his M.S. degree in 2005, and recently, his Ph.D. degree from the Department of Mechatronics Engineering, National Changhua University of Education. He has been using the RFID application system in many fields, such as patrol systems and LTC of the elderly. His research interests include AIOT technology and RFID applications. (201761000018@jmu.edu.cn) 